\title{
„Future of Anticoagulation Initiative" stellt fest: Vorhofflimmern ist unterdiagnostiziert
}

\begin{abstract}
Öffentlichkeit und Patienten mehr für Vorhofflimmern und die Folgen sensibilisieren, frühere Diagnose, bessere Behandlung und wirksamere Schlaganfallprävention anstreben - auf diesen Nenner lassen sich die Empfehlungen eines europäischen Experten-Panels der "The Future of Anticoagulation Initiative" bringen. Sie wurde auf dem EHRA-Kongress in Mailand vorgestellt.
\end{abstract}

V orhofflimmern (VF) hat eine Prävalenz von 1 bis $2 \%$ und ca. $18 \%$ bei über 85-Jährigen. Aufgrund der Altersentwicklung wird die Prävalenz im Jahr 2050 auf 3,3\% geschätzt. Die Anzahl der Patienten in Europa wird dann von 9 auf 16 Millionen ansteigen. VF-Patienten weisen ein fünffach erhöhtes Schlaganfallrisiko auf, etwa jeder 5. Schlaganfall geht auf VF zurück. VF-verursachte Schlaganfälle sind oft schwerwiegend.

\section{Screening empfohlen}

Vor diesem Hintergrund hat das Unternehmen Daiichi-Sankyo das britische Forschungsinstitut „RAND Europe in Cambridge" beauftragt, das VF-Management in sechs EU-Ländern zu analysieren und künftige Entwicklungen aufzuzeigen. Aus Deutschland waren die Professoren Andreas Götte, Paderborn, Reinhold Kreutz, Berlin, und Hans-Christoph Diener, Essen, beteiligt. VF kann asymptomatisch sein und wird unterdiagnostiziert, so der Report. Eine Pulskontrolle bei jedem Arztbesuch, ggf. gefolgt von einem EKG, würde Abhilfe schaffen und wird von der „European Society of Cardiology“ (ESC) als Screeningmaßnahme empfohlen.

Neben der Wiederherstellung des Sinusrhythmus steht die Schlaganfallprophylaxe im Vordergrund. Einsetzbar sind Vitamin-K-Antagonisten sowie neue orale Antikoagulanzien. Letztere gehen mit einem deutlich geringeren Blutungsrisiko einher, wie Götte darlegte. Wie Registerdaten zeigen, werden lei-

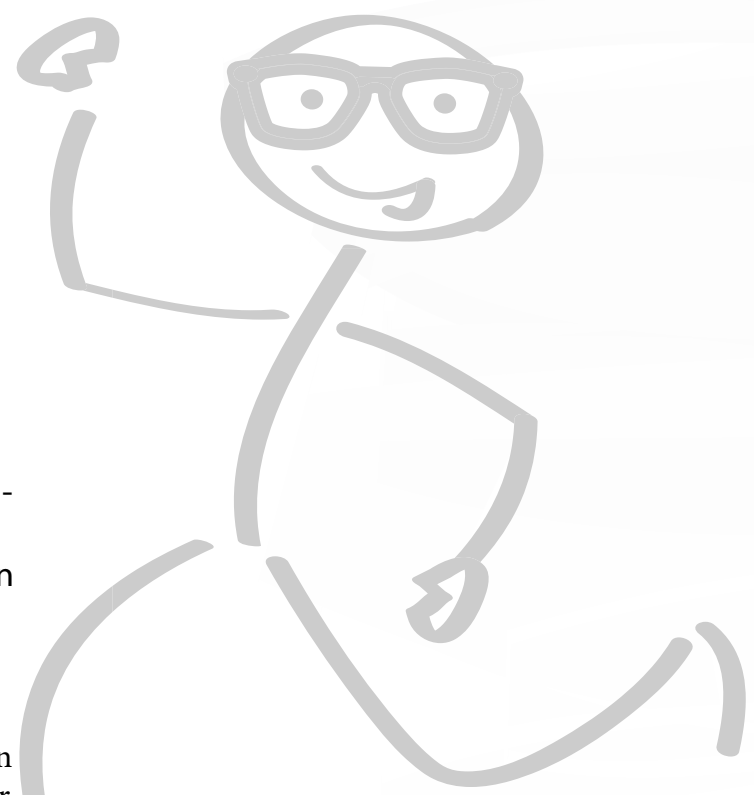

der viele Vorhofflimmern-Patienten mit Antikoagulanzien über- oder untertherapiert.

Vor Therapiebeginn sollte unbedingt der CHA2DS2-VASc-Score bestimmt werden, so Götte. Ein Score von 0 ist keine Therapieindikation. Die Antikoagulation bei einem Score von 1 wird diskutiert, die ESC empfiehlt sie bereits. Bei einem Score von 2, 4 und 6 beträgt das jährliche Insultrisiko 2,3\%, $4 \%$ bzw. 9,8\%. Spätestens hier sollte jeder Patient antikoaguliert werden.

$(D E)$

Quelle: EHRA, Europace Cardiostim,

Media\&Expert Press Conference, Mailand, 21-

24.06.2015 (Veranstalter: Daiichi-Sankyo)

\section{Kabelloser Miniaturschnittmacher zeigt gute Performance}

Die Schrittmacher-Therapie mit einem kabellosen Miniatur-Device ist erfolgreich und komplikationsarm, so ein erster Bericht der "Micra Transcatheter Pacing Study" nach Implantation bei 140 Patienten.

E in permanenter Schrittmacher ist die einzig wirksame The- rapie symptomatischer Bradykardien. Konventionelle transvenöse Schrittmacher führen in 7 bis $12 \%$ zu Komplikationen, manche Patienten müssen reoperiert werden. Einen Fortschritt versprechen neue Miniatur-Schrittmacher, die via Katheter in der Herzkammer platziert werden und ohne Kabel auskommen. Gewicht und Größe dieser Devices sind um ein Zehntel kleiner als bei herkömmlichen Geräten.

Die „Micra Transcatheter Pacing Study Group“ um Dr. Philippe Ritter aus Bordeaux berichtete nun beim EHRA-Europace-Kongress die ersten Sicherheits- und Wirksamkeitsdaten nach Behandlung von bisher 140 Patienten.

\section{Bei 140 Patienten erfolgreich implantiert}

An der weltweiten Studie, die über 700 Patienten einschließen wird, beteiligen sich 23 Zentren, die Patienten mit Klasse 1 und 2-Indikationen für ein ventrikuläres Pacing erstmals mit einem Schrittmacher versorgten.

Die Implantation war zu 100\% erfolgreich. Das Implantat wurde zu 77\% am Apex, zu 22\% am Septum befestigt. Der Eingriff dauerte im Schnitt 37 Minuten. Ernste Komplikationen traten bei 5,7\% der Patienten auf, darunter fünf Rhythmuskomplikationen (Vorhofflimmern, ventrikuläre Tachykardie, Rechtsschenkelblock, transienter AV-Block) und je einmal Myokardinfarkt, Perikardeffusion (ohne Tamponade) und Perikarditis. Zwei Patienten hatten einen längeren Klinikaufenthalt. Es kam zu keinen Todesfällen, unvorhergesehenen Komplikationen, Infektionen, Dislokationen, Reoperationen oder telemetrischen Komplikationen.

Die elektrische Performance sei exzellent und stabil nach bisher bis zu drei Monaten, so Ritter. Die Lebenszeit der Batterie gab er vorläufig mit etwa zwölf Jahren an. 